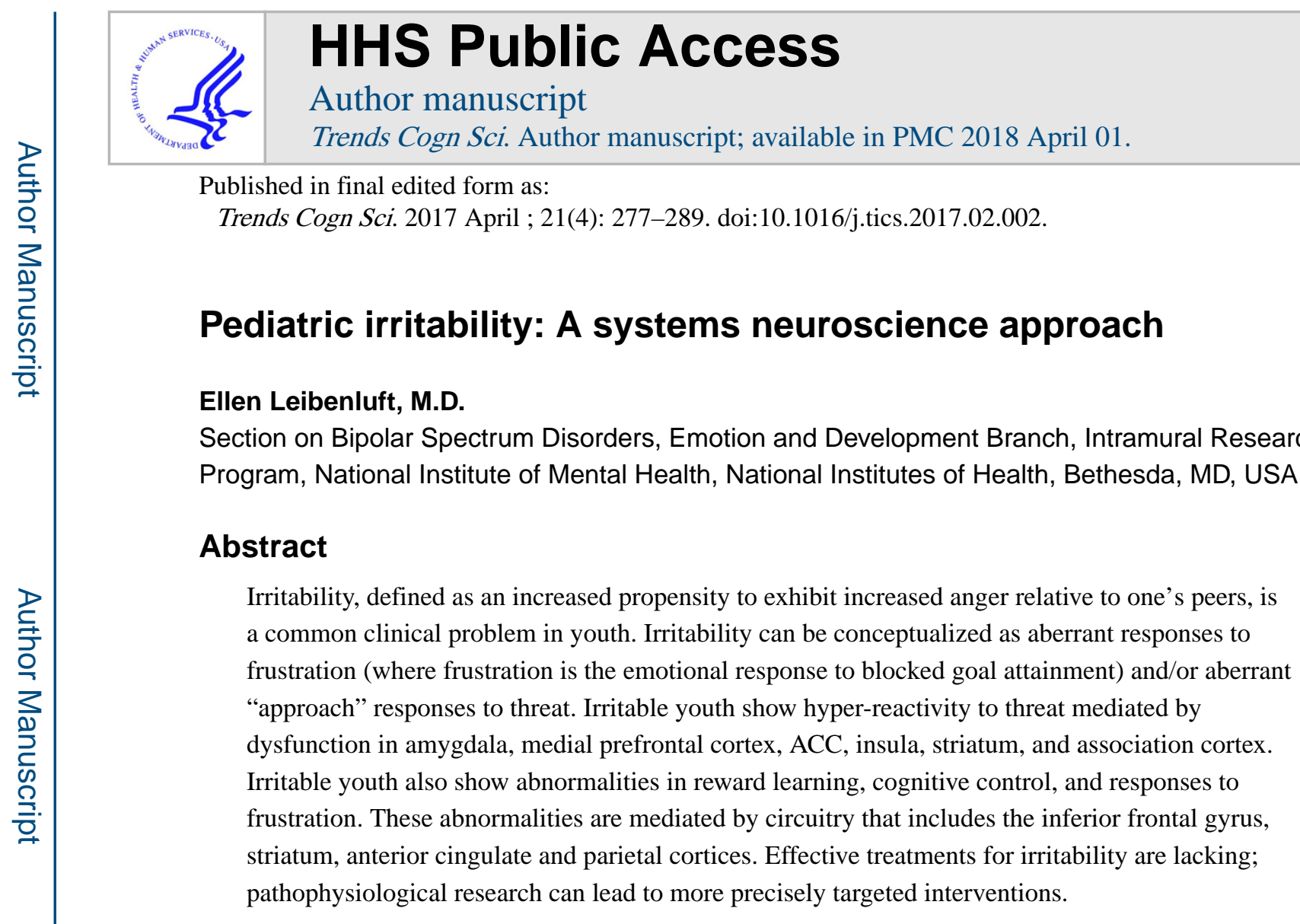

\title{
Keywords
}

irritability; aggression; reward; threat; frustration; anger

\section{Irritability in children}

Irritability (see Glossary) can be defined as an increased propensity to exhibit anger relative to one's peers. Recently, this clinical problem has become the focus of considerable research interest in child psychiatry and clinical neuroscience [1]. This interest stems from recognition of irritability's clinical importance, given that it is one of the most common reasons children present for mental health care [2]. Reflecting this, irritability is the primary feature of a new diagnosis in DSM-5, Disruptive Mood Dysregulation Disorder (DMDD) [3]. Moreover, irritability is prominent in other childhood psychiatric illnesses, including oppositional defiant disorder, anxiety disorders, attention deficit hyperactivity disorder (ADHD), post-traumatic stress disorder, conduct disorder, major depressive disorder, bipolar disorder, and autism spectrum disorders.

Youth with DMDD suffer significant impairment and often require multiple clinical interventions (e.g., medication, school placement, individual and family psychotherapies) to function adequately at home and school [4,5]. However, the efficacy of commonly-used

*Correspondence: leibs@mail.nih.gov.

Publisher's Disclaimer: This is a PDF file of an unedited manuscript that has been accepted for publication. As a service to our customers we are providing this early version of the manuscript. The manuscript will undergo copyediting, typesetting, and review of the resulting proof before it is published in its final citable form. Please note that during the production process errors may be discovered which could affect the content, and all legal disclaimers that apply to the journal pertain. 
treatments for irritability is limited or, in some instances, unknown. Most such treatments are not designed to target irritability specifically and none are based on an understanding of the relevant neurobiology (for a promising avenue that could be an exception, see [6]. Recent work has yielded much information about the presentation, course, and impact of irritability in youth (Box 1). The next challenge is clear: to elucidate the neural mechanisms of irritability in order to guide the development of novel interventions. Pathophysiological studies targeting irritability specifically remain relatively rare, but there is a considerable foundation of work on related phenotypes, such as reactive aggression (Box 2). Within the realm of psychopathology, irritability is a relatively tractable research target because it is an evoked response, and hence can be modeled in animals and studied in real time during neuroimaging. Since research on neural mechanisms of irritability is relatively nascent and rapidly evolving, it is important for investigators to specify neuroscientific conceptualizations to guide research, highlight areas where emerging data warrant followup, and discuss approaches to developing promising research paradigms. These will be the goals of this review, with an emphasis on studies that include clinically impaired youth.

\section{Box 1}

\section{Clinical presentation, longitudinal course, and genetic epidemiology}

In DSM-5, the diagnostic criteria for Disruptive Mood Dysregulation Disorder (DMDD) require both tonic and phasic irritability. Phasic irritability, which is usually the most salient clinical feature, can be operationalized as temper outbursts that are developmentally inappropriate in frequency and severity and that usually occur in response to frustration. Most outbursts are verbal but most irritable children also have some outbursts that involve mild physical involvement or even physical aggression. In addition to outbursts, DMDD requires tonic irritability i.e., between-outburst mood that is angry most days, most of the time. Arguably, the research described in this review is most germane to phasic irritability, which can be evoked using frustration paradigms and whose time course and phenomenology is more akin to the evoked responses of fMRI paradigms than is tonic irritability. Since tonic irritability is a long-lasting mood (rather than a brief emotion linked to a stimulus), it is more challenging to model in the scanner. Importantly, the extent to which phasic and tonic irritability are distinct phenomena in terms of clinical presentation, longitudinal course, or treatment response remains unclear [81, 82], and studies have not yet undertaken the complex task of attempting to dissociate their pathophysiology.

In healthy youth, the frequency of temper outbursts peaks in the preschool years and then gradually declines, in tandem with prefrontal cortical development $[81,83]$. While temper loss is common in preschoolers, normative and non-normative tantrums can be distinguished using a parent-completed developmentally sensitive questionnaire [84]. Further, non-normative temper loss predicts the onset of mood and behavioral symptoms and disorders 16 months later [85]. In infants, it is possible to identify individual differences in responses to frustration; maternal, rather than lab-based, measures, are better predictors of irritability in toddlerhood $[86,87]$. A new wave of lab-based and observational studies in infants using novel techniques may have more predictive power. 
Finally, normal adolescence is typically associated with a small upsurge of both tonic and phasic anger proneness [81,88].

Irritable youth (i.e., those with increased tonic and phasic anger, relative to their peers) are at elevated risk to develop anxiety, unipolar depressive disorders, and/or suicidality in adolescence and early adulthood, and to have decreased educational and income attainment [89]. Twin studies suggest that the heritability of irritability is approximately 0.4-0.6 [90-92]. Longitudinal associations between irritability and depression/anxiety are partially genetically mediated, and the proportion of the variance in irritability accounted for by genetic factors differs with gender and age [90-92]. Environmental factors also play a significant role in the etiology of irritability, as evidenced by the efficacy of parenting interventions in decreasing child irritability, especially in young children [93].

\section{Box 2}

\section{Clinical phenotypes relevant to research on irritability}

In DSM-5, the pediatric diagnostic categories most germane to the study of irritability are DMDD and oppositional defiant disorder (ODD). Research on irritability is also wellsuited to a dimensional approach. This is because irritability is distributed continuously across the population and because the precise cut-point differentiating typical from atypical irritability is unclear. Moreover, irritability occurs in many diagnoses (anxiety, ADHD), and a continuous approach facilitates quantification of multiple symptoms simultaneously. Most youth with DMDD also meet diagnostic criteria for ADHD, and many meet diagnostic criteria for an anxiety disorder [4,5]. Conversely, youth diagnosed with ADHD or anxiety exhibit high rates of irritability $[64,94]$. This co-occurrence among irritability, anxiety, and ADHD may reflect the contribution of both dysfunctional threat processing and attentional dyscontrol to the pathophysiology of irritability. This co-occurrence also raises an important question regarding the extent to which irritability manifesting in different diagnostic contexts is mediated by overlapping or separable neural circuitry (see main text).

As noted in Box 1, irritable youth may exhibit aggressive behavior during a temper outburst. The brain mechanisms of aggression have received considerable research attention, and studies of reactive aggression are particularly germane to irritability. Reactive or affective aggression is an intense, impulsive emotional response to anger, usually precipitated by frustration or threat [95]. Thus, reactive aggression can be seen as the most extreme behavioral manifestation of irritability. Another type of aggression has been termed proactive or instrumental. This type of aggression is designed to accomplish a goal (e.g., steal money) and is seen in psychopathic individuals and those with callousunemotional traits. The pathophysiology of reactive and proactive aggression appears to differ (see main text). As clinical syndromes, reactive aggression is more common than proactive aggression and often exists without co-occurring proactive aggression, while proactive aggression almost universally co-occurs with reactive aggression. This complex clinical pattern complicates the application of findings in the aggression literature to 
questions about the pathophysiology of irritability. However, work differentiating aggression in psychopathic adults vs. those with externalizing traits [96], or in youth with disruptive behavior disorders with or without callous-unemotional traits e.g., ([35, 44]; see main text), is helpful in differentiating the pathophysiology of reactive from proactive aggression, and thus in advancing research on irritability.

\section{The neuroscience of irritability}

Translational models of irritability focus on two closely related but separable processes: dysfunctional threat and reward processing [1] (Figure 1, Key Figure). Thus, one hypothesis suggests that irritability reflects dysfunction in the amygdala-hypothalamic-PAG threat response circuitry, such that approach responses occur in contexts where the normative response would be freezing or flight [7]. The common co-occurrence of anxiety and irritability, as well as the genetic and longitudinal links between them (Boxes 1,2), may thus represent vacillation between abnormal avoid and approach responses to threat, reflecting disequilibrium in the mediating circuitry that is, in part, genetically mediated. As discussed below, data in irritable youth and related phenotypes demonstrate abnormalities in threat processing specifically, and in social information processing more broadly. Also as discussed below, this theory has been probed using threat stimuli that are relatively simple, such as angry faces, or that involve more complex social interaction paradigms.

The second hypothesis suggests associations between irritability and abnormal reward processing, specifically in the form of aberrant responses to frustrative non-reward (FNR). In landmark work, Amsel defined FNR as the psychological state induced by the failure to receive a reward that a rodent has been conditioned to expect. Amsel showed that FNR is associated with increased motor activity and aggression [8]. Research has documented FNR responses in non-human primates and humans [9-11] (Figure 1). This work is also clinically relevant, since temper outbursts in irritable children often occur in response to frustration. Taken together, these basic and clinical data suggest that pathological temper outbursts in children may reflect FNR responses that are abnormal in their intensity, duration, and/or the strength of the provocation required to induce them. As detailed below, imaging paradigms that assess fundamental components of reward learning, or that induce FNR during neuroimaging, can test this hypothesis. In irritable youth and related phenotypes, such studies find abnormalities in reward learning, cognitive control processes that mediate reward learning, and responses to FNR.

Importantly, while stated as dissociable hypotheses, the threat and reward formulations of irritability are inextricably intertwined both conceptually and empirically. In an extension of Amsel's work, one study demonstrated an interaction between FNR and threat processing, in that frustrated mice exhibited increased aggressive responses in an intruder paradigm ([12], Figure 1). Indeed, based on other rodent research, Gray [13] proposed the "fear=frustration" hypothesis, suggesting that frustration is processed as a threat and responses to both are mediated by the behavioral inhibition system. A similar formulation emerges from research in humans i.e., in paradigms that model competitive games, being ostracized or treated unfairly has been viewed by investigators as either a frustrating or a threatening experience 
$[14,15]$. Consistent with Gray's formulation, some research [16] suggests that regions mediating threat processing and reactive aggression (i.e., amygdala, PAG, insula and dorsal anterior cingulate cortex) are engaged during blocked reward. In contrast, other studies $[17,18]$ found competitive processes between regions responding to threat of shock and those engaged in decision-making about a monetary reward. However, this work is only beginning. Important questions suggested by these conflicting data include the impact of the features of the threatening or rewarding stimulus (simple vs. complex, social vs. non-social) on the circuitry engaged, as well as whether distinct clinical phenotypes of irritability have a stronger association with reward- vs. threat-related dysfunction.

\section{Social information processing in irritable youth}

Irritability in youth usually manifests in social situations, suggesting that irritable youth may experience such situations differently than do non-irritable youth. Indeed, multiple lines of research indicate that irritable youth have social information processing deficits.

The most consistent line of research on social information processing deficits in irritable youth finds perturbed threat processing. One body of research assesses irritable youth's responses to relatively simple, iconic social signals, particularly angry faces (e.g., Figure 2), whereas other studies use paradigms that model more complex social processes. In the face emotion processing literature, paradigms vary in the duration of stimulus exposure and in the cognitive processes engaged. One set of studies uses paradigms such as the dot probe task to assess individual differences in attention-related threat bias i.e., the tendency to orient preferentially and rapidly to an angry vs. neutral face. In these studies, the face stimulus is presented for a relatively brief period (e.g., 17-1250 ms) and serves as a distractor. Dot probe studies in both clinical and community samples of irritable youth find an attention bias toward angry faces $[19,20]$. Similar findings manifest in youth with anxiety disorders and have given rise to a treatment (attention bias modification training) designed to decrease anxiety by reversing the threat bias [21,22]. Shared deficits in attention orienting provide one possible pathophysiological basis for the cross-sectional, genetic, and longitudinal associations between anxiety and irritability. To date, there are no attention bias modification training trials in irritable youth, and no fMRI studies in irritable youth that use an attention orienting task. However, studies of the dot probe task in anxious youth most consistently find abnormalities in amygdala-ventromedial prefrontal cortex connectivity [23-25].

In other face emotion processing paradigms, participants are asked to process a specified aspect of the stimulus. Typically, the face is shown for approximately $2000-4000 \mathrm{~ms}$ and the subject labels either the emotion (explicit face emotion processing) or another facial feature, such as gender (implicit processing). Given the higher-order cognitive processing involved in emotional labeling, explicit processing tasks would be expected to engage association cortex, whereas some evidence suggests that implicit face processing tasks activate the amygdala more reliably [26]. On explicit processing tasks, irritable youth show behavioral deficits, in that they label face emotions inaccurately $[27,28]$. They also judge neutral faces as more hostile than do healthy youth, whether the stimulus duration is brief (i.e., $200 \mathrm{~ms}$ ) [29] or not (i.e., $4000 \mathrm{~ms}$ ) [30]. Irritable youth show amygdala, ventral visual stream, and 
association cortex dysfunction during explicit face processing [30-32], although the nature of the amygdala dysfunction (hypo- vs. hyperactivation) varies across tasks.

As noted above, compared to explicit face processing tasks, implicit tasks may be more efficient at eliciting between-group differences in amygdala activity because they are less likely to elicit PFC engagement, and, hence, possible amygdala inhibition [26]. Reactive aggression can be viewed as the most extreme behavioral manifestation of irritability. Thus, it is interesting to note that studies of youth recruited for reactive aggression or irritability find amygdala hyperactivation to implicit processing of negatively valenced faces [33-35]. In contrast, subjects in these studies who exhibit proactive aggression show amygdala hypoactivation on such paradigms [34,35]. This is consistent with the formulation that youth with irritability or reactive aggression have impulsive "hot" emotional responses to threat, whereas those with proactive aggression process threat in a more considered, "cold" fashion. fMRI data acquired at rest or during an implicit face processing task have also found associations between youth irritability and aberrant activity in other regions, including insula, cingulate, and striatum, but such findings bear replication [33,36,37].

An important question is whether the neural mechanisms mediating irritability vary with differences in clinical phenotype, since irritability can occur with or without various other clinical problems. This includes reactive aggression, and pediatric mental disorders that include DMDD, ADHD, anxiety disorders, and pediatric bipolar disorder (BD). Is the pathophysiology of irritability similar across these clinical contexts? Two studies address this question using face emotion processing tasks (one implicit, [37], one explicit [32]). Data from both studies suggest that clinical context moderates the neural correlates of irritability, albeit in somewhat different ways. In the study using explicit face emotion labeling [32], diagnosis was the key parameter influencing neural correlates: children with BD and those with DMDD differed in amygdala and ventral visual stream activity while labeling face emotions. However, in the study involving implicit face emotion processing [37] (Figure 2), severity on an anxiety dimension was the key parameter. Specifically, in the latter study, independent of diagnosis (ADHD, anxiety, DMDD, or none), youth with high levels of both irritability and anxiety tended to have low amygdala-medial PFC connectivity when processing angry faces implicitly, whereas those with anxiety alone tended to have high connectivity. These two studies $[32,37]$ differ in many aspects, including task and the diagnoses compared (of note, $\mathrm{BD}$ is a particularly distinct phenotype that is highly heritable [38]); clearly this important question requires further study.

While paradigms that use face stimuli have the advantage of relatively tight experimental control, they lack ecological validity. To increase the latter, investigators have devised fMRI paradigms designed to model reactive aggression. In paradigms such as the Ultimatum Game [39] or Point Subtraction Aggression Paradigm [40], subjects are told that they are playing a computer game with another person, although the actions of the other "person" are programmed by the investigator. These paradigms assess correlates of reactive aggression (again, an extreme behavioral expression of irritability), by quantifying the extent to which a subject engages in uncooperative or retaliatory behavior in response to the other "player" stealing money, making an unfair offer, or rejecting an ambiguous offer. 
While few studies apply these paradigms to children with psychopathology, the results that exist are intriguing. Children recruited for either irritability or reactive aggression show an increase in uncooperative or aggressive behaviors toward the other "player," including in response to ambiguous behavior [41-44]. Thus, irritable or aggressive youth have a "hostile interpretation bias" which can be expressed in diverse contexts and studied from multiple scientific perspectives. On simpler paradigms, this bias could lead irritable children to label ambiguous faces as angry, while on more complex paradigms, this bias could cause irritable youth to interpret ambiguous peer behavior as threatening [29,45]. Studies suggest that aggressive responding on complex paradigms such as the Point Subtraction Aggression Paradigm or Ultimatum Game is associated with aberrant activity in a threat-mediating circuit including the amygdala, periaqueductal gray, ventromedial PFC, striatum and insula, along with decreased amygdala-ventromedial PFC connectivity $[43,44]$. The ventromedial PFC findings are consistent with an extensive literature suggesting that ventromedial PFC lesions in adult- or childhood cause irritability and aggression [46-48].

In sum, youth with irritability and related phenotypes show hyperreactivity to negatively valenced social stimuli. This manifests as biased orienting to angry faces and as a bias toward interpreting ambiguous social signals as more threatening than do healthy youth. These biases are associated with amygdala dysfunction, most commonly hyperactivation during implicit processing of negatively valenced faces and decreased connectivity with medial prefrontal cortex. In addition, such biases may be associated with abnormal activation in the ACC, insula, striatum, and association cortex.

\section{Reward processing in irritable youth}

The FNR model posits that animals respond to the omission of an expected reward with increased activity and aggression; this raises the possibility that irritable youth's outbursts represent exaggerated FNR responses. Reward learning deficits in irritable youth could decrease their ability to respond appropriately to reward contingencies, increasing both the probability of frustrating experiences and maladaptive responses to them. Consistent with these possibilities, studies suggest that irritable youth have deficits in passive avoidance and reversal learning, and fMRI studies find associated dysfunction in regions that mediate these psychological processes, including ventromedial PFC, striatum, insula, ACC, and inferior frontal gyrus (iFG) [49-53]. Computational modeling suggests that a decreased ability to represent expected value information in some of these regions, including insula, $\mathrm{iFG}$, caudate, and ventromedial PFC, may contribute to impaired decision-making in irritable youth $[51,52,54]$.

Beyond difficulty representing expected value, deficient reward learning could arise from impairments in other cognitive control functions. These include the ability to withhold a prepotent response, use working memory to deploy behavior flexibly in complex scenarios, or register and change stimulus-response mappings after making an error. In fact, an extensive literature links pediatric aggression to these and other components of cognitive control [5558]. While these studies generally do not differentiate reactive from proactive aggression, one study did find a specific association between reactive aggression and deficits in sustained attention and set-shifting [59]. Further evidence of impaired cognitive control in 
irritable youth arises from imaging and electrophysiology studies. For example, prior studies demonstrate an association between inhibitory control deficits in irritable youth and reduced cognitive control capacities. The latter is reflected in the amplitude of event-related potential components, such as N2 and P3 amplitudes [60-62].

Importantly, irritability, cognitive control deficits, and decision-making abnormalities are all common in youth with ADHD. Irritability is elevated in both community and clinic samples of ADHD, with a 10-fold increase in one large community sample and a range of 24-50\% in clinic samples $[63,64]$. While the mechanisms that support associations between ADHD and irritability remain unclear, there is relevant research. A recent study that subtyped patients with ADHD ( $n=437$ ) using resting state fMRI, peripheral physiological methods, and clinical outcome detected an irritable subtype that showed weak parasympathetic response to negative emotional stimuli, reduced amygdala-insula connectivity, and relatively poor outcome [65]. Regarding cognitive control deficits in ADHD, working memory impairment is among the most common [66]. Working memory is thought to play an important part in emotion regulation, in part through facilitating cognitive reappraisal and adaptive responding to changing environmental contexts [67]. During an n-back working memory task that included emotional stimuli, youth with ADHD showed abnormal engagement in multiple PFC regions and striatum [68]. In youth with ADHD, working memory deficits may also contribute to suboptimal decision-making in emotional contexts [69]. Indeed, a number of studies in youth with ADHD find impulsive decision-making, assessed using delayed discounting tasks; impulsive responding to threat or frustration is characteristic of irritability and reactive aggression. On delayed discounting tasks, youth with ADHD show a preference for small, immediate rewards over large, delayed rewards [64,70]. Studies have found associations between such impulsive decision-making and both nucleus accumbens-PFC connectivity and amygdala hyperactivity in youth with ADHD [71,72].

As noted above, studies of perturbed threat processing in irritable youth rely on diverse forms of social stimuli, including both simple iconic images and more complex social game paradigms. Similar diversity applies in studies of perturbed reward processing in irritable youth. Thus, some studies use relatively simple paradigms to assess basic reward learning or cognitive control functions in irritable youth, while others use more complex paradigms that model FNR directly. In the latter, frustration is induced by rigged games or unsolvable puzzles that withhold an expected reward, and investigators report associations between irritability and the degree of frustration induced by the task [62,73-75]. These studies typically find associations between irritability and dysfunction in regions mediating executive attention and reward processing. For example, in a study using functional near infrared spectroscopy imaging in a sample of preschool children, decreased lateral PFC activity was associated with increased frustration tolerance [76]. In older youth, studies of frustration during fMRI or magnetoencephalography show associations between irritability and ACC, striatal, medial PFC, parietal and amygdala activity [73-75, 77] (Figure 3). Somewhat unexpectedly, in two fMRI studies irritability is associated with decreased amygdala activation during frustration [74,75]. However, the time course of the frustrative response can be prolonged, complicating the interpretation of baseline activity. The design of such paradigms is also challenging because of potential order effects (frustration cannot be "turned off" immediately), and because the frustration must be potent enough to be 
effective but not so potent that the child can't tolerate it and/or moves during scanning. In addition, such tasks often include an element of deception which requires careful ethical consideration and can also be logistically difficult. Thus, frustration tasks are challenging to implement and engage a number of complex psychological phenomena. Nonetheless, the ability to evoke FNR directly in the scanner is an important tool in the effort to elucidate the neural mechanisms of pediatric irritability.

Ironically, Amsel's FNR studies are motivating human research currently, but there have been relatively few recent studies extending his seminal work in rodents. This is unfortunate because, arguably, the most effective translational work in psychiatry has been in anxiety disorders and substance abuse [78,79], and these disorders have salient commonalities with irritability. Specifically, these three phenotypes can all be conceptualized as evoked responses, making it feasible to design parallel paradigms in humans and animals that model the relevant psychological processes. In the case of irritability, one important line of research would be to identify mouse strains that are highly reactive to FNR e.g., in terms of aggression and motor activity (see Outstanding Questions box). This would enable more precise mapping of circuits that are activated differentially in irritable animals. The assumption is that interstrain variability will be larger than intrastrain variability although, should the latter be more prominent, this would provide an excellent opportunity to focus on individual differences, as is done in research on human psychopathology.

\section{Outstanding Questions Box}

- In rodents, is there interstrain and intrastrain variability in the motor activity and aggression stimulated by frustrative non-reward? What differences in circuitry function mediate such variability?

- How does the circuitry mediating irritability differ across clinical contexts (e.g., in the presence or absence of anxiety, reactive aggression, attention deficit hyperactivity disorder)?

- How do threat-based and reward-based pathways to irritability interact neurally and clinically? Can one define subtypes of irritable youth based on the mediating mechanism and, if so, what are the treatment implications of such subtyping?

- How does dysfunction in threat reward circuitry differ in irritable youth (who frequently also have anxiety disorders) vs. those with anxiety alone?

- Reactive aggression can be seen as the most extreme behavioral manifestation of irritability. Are the neural mechanisms mediating these two clinicallydefined phenotypes on a continuum?

- Deficits in both inhibition and working memory have been implicated in the pathophysiology of irritability. What cognitive control deficits are most relevant to the pathophysiology of irritability?

- Are irritable youth hyper-reactive to non-social threat, as well as social threat? 
- If the hyper-reactivity of irritable youth to social threat is ameliorated by computer-based implicit training, would this intervention be effective in decreasing irritability?

- At baseline, youth with irritability have cognitive control deficits. Are such deficits exacerbated in the context of frustrative non-reward? Relatedly, what is the precise nature of the attentional dysfunction that irritable youth exhibit when frustrated?

- What deficits in the computational mechanisms mediating reward learning are important in irritability?

\section{Concluding Remarks}

Motivated by its clinical importance, researchers have begun to elucidate the neural correlates of pediatric irritability. In irritable youth, researchers have identified abnormalities in both threat and reward processing. Studies consistently show hyper-reactivity toward threat in irritable youth. Specific findings include an attentional bias toward threat, a tendency to view ambiguous faces as angry, and a propensity to interpret a competitor's ambiguous actions as hostile. These abnormalities are associated with dysfunction in the circuitry mediating threat processing (amygdala, periaqueductal grey, ventromedial PFC) as well as other regions, depending on the behavioral deficit. Irritable youth also show abnormalities on paradigms that model frustrative non-reward; those that assess more basic aspects of reward processing; and those that probe cognitive control functions which impact on reward processing. Such deficits are associated with dysfunction in the well-defined circuitry mediating reward learning, including ventromedial PFC, insula, ACC, striatum, and inferior frontal gyrus. Paradigms modeling FNR also evoke deficits in executive attention and parietal dysfunction in irritable youth. Impaired cognitive control, particularly during inhibition, is present in irritable youth; such deficits, as well as impulsive decision-making, are present in youth with ADHD, who are at particularly high risk for irritability.

Of course, since neurally-based research on irritability is relatively new, there are many questions to be addressed (see Outstanding Questions box). On the broadest level, it is important to determine how threat-based and reward-based pathways to irritability interact both neurally and clinically, and how the neural circuitry mediating irritability varies across clinical contexts. The former question could be addressed by paradigms that examine associations of irritability with threat and reward processing at baseline, when a threatening stimulus is presented in the context of a rewarding stimulus, and vice versa. It is unknown whether, among irritable youth, there are distinguishable clinical subtypes in which dysfunctional reward or threat processing is more prominent; addressing this question is consistent with the new emphasis on personalized approaches to medical diagnosis and treatment [80]. Similarly, neural specificity within the broad phenotype of irritability can be probed by studies examining whether the circuitry mediating irritability varies across clinical context (e.g., in the presence of reactive aggression, across different diagnoses, etc.), as well as by studies examining how irritability and other traits (e.g., anxiety) [37] interact in their impact on brain function. 
Beyond these broad research questions, more specific directions for future research are suggested by the preceding review. To advance research on social information processing in youth with irritability or reactive aggression, it is important to integrate work on these two related phenotypes through better behavioral phenotyping in youth recruited for irritability and better assessment of emotional symptoms in youth recruited for reactive aggression. Few studies of youth recruited for irritability use paradigms designed to study individual differences in "approach" behavior in response to threat, or in the mediating circuitry; the use of such paradigms would also bridge work with reactive aggression. To elucidate reward processing dysfunction associated with irritability, researchers could continue and expand the use of computational approaches to define precisely the relevant learning deficits. Finally, there are relatively few studies of cognitive control function in irritable children at baseline and during frustration; the results of such studies could guide the development of novel interventions.

Understanding the pathophysiology of any psychiatric symptom or syndrome is challenging, and this is particularly true in children, where multiple problems typically co-occur.

Irritability is an important focus for research because it is common and associated with both current and long-term impairment, and because few effective treatments exist. Thus, there is a public health imperative to define the neural mechanisms mediating irritability and to use this knowledge to guide the development of novel interventions. Fortunately, there are reasons to believe that irritability may be a relatively tractable target for research, and considerable progress has been made in a relatively short period of time. Further work that brings relief to affected children and their families would be most welcome.

\section{Acknowledgments}

The author thanks Daniel Pine, MD for comments on manuscript drafts and helpful conversations; Alexandra Roule and Gretchen Perhamus for editorial assistance; past and present members of the Emotion and Development Branch for their scientific contributions; and our patients and their families for their invaluable contributions to the research.

Supported by the NIMH Intramural Research Program, conducted under NIH Clinical Study Protocols 15-M-0182 (ClinicalTrials.gov identifier: NCT0531893), 02-M-0021 (ClinicalTrials.gov identifier: NCT00025935), and 00M-0198 (ClinicalTrials.gov identifier: NCT00006177).

\section{Glossary}

\section{anxiety disorders}

a group of related psychiatric diagnoses characterized by an abnormally heightened tendency to avoid threatening stimuli, and by impairment related to this tendency

\section{attention deficit hyperactivity disorder (ADHD)}

DSM-5 diagnosis characterized by persistent inattention and/or impulsivity-hyperactivity that interferes with function or development

\section{cognitive control}

psychological processes that facilitate flexible behavior and attention deployment in response to changing goals and environmental circumstances

disruptive mood dysregulation disorder (DMDD) 
childhood diagnosis, new in DSM-5, characterized by severe, impairing, chronic irritability (both phasic and tonic)

\section{Diagnostic and Statistical Manual Fifth Edition (DSM-5)}

the compilation of standardized criteria for psychiatric diagnoses in the United States, published by the American Psychiatric Association

ecological validity

the degree to which research findings are likely to generalize to real-world situations

explicit face emotion processing paradigm

cognitive task in which the subject is asked to label the emotion on a face

frustration

emotional response to blocked goal attainment

frustrative non-reward (FNR)

as per Amsel (see text), the psychological state induced by the failure to receive a reward that an organism has been conditioned to expect

hostile interpretation bias

the tendency to interpret ambiguous social stimuli as hostile. The stimuli can be either simple (e.g., faces) or complex (e.g., vignettes, or behavior; in this case the commonly-used term is hostile attribution bias)

\section{implicit face emotion processing paradigm}

cognitive task in which the subject is asked to attend to a facial feature other than the emotional display (e.g., gender of the face)

irritability

an increased propensity to experience anger, relative to one's peers

oppositional defiant disorder (ODD)

childhood diagnosis in DSM-5 characterized by impairment due to chronic irritability and/or oppositional headstrong behavior. Compared to DMDD, the severity of the irritability required for ODD is lower.

phasic irritability

irritability manifest as temper outbursts that are developmentally inappropriate in frequency and severity

proactive aggression

aggression designed to accomplish a goal (e.g., theft), generally exhibited by individuals with psychopathy and/or a tendency to respond to emotional stimuli in a callous and unemotional way

reactive aggression 
aggression that occurs as part of an intense, emotional response to anger and is usually

precipitated by frustration or threat

\section{reward}

a stimulus associated with approach behavior

\section{reward learning}

the process by which organisms learn associations between either a stimulus or behavior, on the one hand, and reward or punishment, on the other

\section{threat}

a stimulus associated with avoid behavior

\section{threat bias}

the tendency to orient preferentially and rapidly to a threatening, vs. neutral, stimulus

\section{tonic irritability}

irritability manifest as chronically angry mood

\section{References}

1. Brotman M, et al. Irritability in youth: A translational model. Am J Psychiatry. in press.

2. Peterson BS, et al. Risk factors for presenting problems in child psychiatric emergencies. J Am Acad Child Adolesc Psychiatry. 1996; 35:1162-1173. [PubMed: 8824060]

3. American Psychiatric Association. Diagnostic and Statistical Manual of Mental Disorders: DSM-5. American Psychiatric Publishing; 2013.

4. Axelson D, et al. Examining the proposed disruptive mood dysregulation disorder diagnosis in children in the Longitudinal Assessment of Manic Symptoms study. J Clin Psychiatry. 2012; doi: 10.4088/JCP.12m07674

5. Leibenluft E. Severe mood dysregulation, irritability, and the diagnostic boundaries of bipolar disorder in youth. Am J Psychiatry. 2011; 168:129-142. [PubMed: 21123313]

6. Penton-Voak IS, et al. Increasing recognition of happiness in ambiguous facial expressions reduces anger and aggressive behavior. Psychol Sci. 2013; 24:688-697. [PubMed: 23531485]

7. Blair RJ. The neurobiology of impulsive aggression. J Child Adolesc Psychopharmacol. 2016; doi: 10.1089/cap.2015.0088

8. Amsel A. The role of frustrative nonreward in noncontinuous reward situations. Psychol Bull. 1958; 55:102-119. [PubMed: 13527595]

9. Davenport JW, Thompson CI. The Amsel frustration effect in monkeys. Psychon Sci. 1965; 3:481482.

10. Ryan TJ, Watson P. Frustrative nonreward theory applied to children's behavior. Psychol Bull. 1968; 69:111-125. [PubMed: 4867595]

11. Dollard, J., et al. Frustration and Aggression. Yale University Press; 1939.

12. Burokas A, et al. Operant model of frustrated expected reward in mice. Addict Biol. 2012; 17:770 782. [PubMed: 22264360]

13. Gray, JA. The Psychology of Fear and Stress. Cambridge University Press; 1987.

14. Kawamoto T, et al. Intrapersonal and interpersonal processes of social exclusion. Front Neurosci. 2015; 9:1-11. [PubMed: 25653585]

15. Leary MR, et al. Interpersonal rejection as a determinant of anger and aggression. Pers Soc Psychol Rev. 2006; 10:111-132. [PubMed: 16768650]

16. Yu R, et al. The neural signature of escalating frustration in humans. Cortex. 2014; 54:165-178. [PubMed: 24699035] 
17. Choi JM, et al. Pervasive competition between threat and reward in the brain. Soc Cogn Affect Neurosci. 2014; 9:737-750. [PubMed: 23547242]

18. Choi JM, et al. Counteracting effect of threat on reward enhancements during working memory. Cogn Emot. 2015; 29:1517-1526. [PubMed: 25559397]

19. Salum GA, et al. Association between irritability and bias in attention orienting to threat in children and adolescents. J Child Psychol Psychiatry. in press.

20. Hommer RE, et al. Attention bias to threat faces in severe mood dysregulation. Depress Anxiety. 2014; 31:559-565. [PubMed: 23798350]

21. Pergamin-Hight L, et al. Content specificity of attention bias to threat in anxiety disorders: A metaanalysis. Clin Psychol Rev. 2015; 35:10-18. [PubMed: 25462110]

22. Linetzky M, et al. Quantitative evaluation of the clinical efficacy of attention bias modification treatment for anxiety disorders. Depress Anxiety. 2015; 32:383-391. [PubMed: 25708991]

23. Monk CS, et al. Amygdala and ventrolateral prefrontal cortex activation to masked angry faces in children and adolescents with generalized anxiety disorder. Arch Gen Psychiatry. 2008; 65:568576. [PubMed: 18458208]

24. Hardee JE, et al. Patterns of neural connectivity during an attention bias task moderate associations between early childhood temperament and internalizing symptoms in young adulthood. Biol Psychiatry. 2013; 74:273-279. [PubMed: 23489415]

25. Price RB, et al. Vigilance in the laboratory predicts avoidance in the real world: A dimensional analysis of neural, behavioral, and ecological momentary data in anxious youth. Dev Cogn Neurosci. 2016; 19:128-136. [PubMed: 27010577]

26. Lieberman MD, et al. Putting feelings into words: Affect labeling disrupts amygdala activity in response to affective stimuli. Psychol Sci. 2007; 18:421-428. [PubMed: 17576282]

27. Guyer AE, et al. Specificity of face emotion labeling deficits in childhood psychopathology. J Child Psychol Psychiatry. 2007; 48:863-871. [PubMed: 17714371]

28. Rich BA, et al. Face emotion labeling deficits in children with bipolar disorder and severe mood dysregulation. Dev and Psychopathol. 2008; 20:529-546.

29. Stoddard J, et al. An open pilot study of training hostile interpretation bias to treat Disruptive Mood Dysregulation Disorder. J Child Adolesc Psychopharmacol. 2016; 26:49-57. [PubMed: 26745832]

30. Brotman MA, et al. Amygdala activation during face emotion processing in children with severe mood dysregulation vs. ADHD vs bipolar disorder. Am J Psychiatry. 2010; 167:61-69. [PubMed: 19917597]

31. Thomas LA, et al. Parametric modulation of neural activity by emotion in youth with bipolar disorder, youth with severe mood dysregulation, and healthy volunteers. Arch Gen Psychiatry. 2012; 69:1257-1266. [PubMed: 23026912]

32. Wiggins JL, et al. Neural correlates of irritability in disruptive mood dysregulation and bipolar disorders. Am J Psychiatry. 2016; 173:722-730. [PubMed: 26892942]

33. Thomas LA, et al. Elevated amygdala responses to emotional faces in youths with chronic irritability or bipolar disorder. Neuroimage Clin. 2013; 2:637-645. [PubMed: 23977455]

34. Sebastian CL, et al. Neural responses to fearful eyes in children with conduct problems and varying levels of callous-unemotional traits. Psychol Med. 2014; 44:99-109. [PubMed: 23510564]

35. Viding E, et al. Amygdala response to preattentive masked fear in children with conduct problems: The role of callous-unemotional traits. Am J Psychiatry. 2012; 169:1109-1116. [PubMed: 23032389]

36. Hulvershorn LA, et al. Abnormal amygdala functional connectivity associated with emotional lability in children with attention-deficit/hyperactivity disorder. J Am Acad Child Adolesc Psychiatry. 2014; 53:351-361. [PubMed: 24565362]

37. Stoddard J, et al. Irritability, anxiety, and the neural processes of implicit face emotion processing in youth. JAMA Psychiatry. 2017; 74:95-103. [PubMed: 27902832]

38. McGuffin P, et al. The heritability of bipolar affective disorder and the genetic relationship to unipolar depression. Arch Gen Psychiatry. 2003; 60:497-502. [PubMed: 12742871] 
39. Güth W, et al. An experimental analysis of ultimatum bargaining. J Econ Behav Organ. 1982; 3:367-388.

40. Cherek DR. Effects of smoking different doses of nicotine on human aggressive behavior. Psychopharmacology (Berl). 1981; 75:339-345. [PubMed: 6803276]

41. Northover $\mathrm{C}$, et al. Emotion regulation in adolescent males with attention-deficit hyperactivity disorder: Testing the effects of comorbid conduct disorder. Brain Sci. 2015; 5:369-386. [PubMed: 26371048]

42. Greenwald MK, et al. Teens with heavy prenatal cocaine exposure respond to experimental social provocation with escape not aggression. Neurotoxicol Teratol. 2011; 33:198-204. [PubMed: 20600841]

43. Bubenzer-Busch S, et al. Neural correlates of reactive aggression in children with attention-deficit/ hyperactivity disorder and comorbid disruptive behaviour disorders. Acta Psychiatr Scand. 2016; 133:310-323. [PubMed: 26292852]

44. White SF, et al. Neural correlates of the propensity for retaliatory behavior in youths with disruptive behavior disorders. Am J Psychiatry. 2016; 173:282-290. [PubMed: 26441155]

45. Crick NR, Dodge KA. Social information-processing mechanisms in reactive and proactive aggression. Child Dev. 1996; 67:993-1002. [PubMed: 8706540]

46. Anderson SW, et al. Impairments of emotion and real-world complex behavior following childhood- or adult-onset damage to ventromedial prefrontal cortex. J Int Neuropsychol Soc. 2006; 12:224-235. [PubMed: 16573856]

47. Berlin HA, et al. Impulsivity, time perception, emotion and reinforcement sensitivity in patients with orbitofrontal cortex lesions. Brain. 2004; 127:1108-1126. [PubMed: 14985269]

48. Grafman J, et al. Frontal lobe injuries, violence, and aggression: a report of the Vietnam Head Injury Study. Neurology. 1996; 46:1231-1238. [PubMed: 8628458]

49. Adleman NE, et al. Neural correlates of reversal learning in severe mood dysregulation and pediatric bipolar disorder. J Am Acad Child Adolesc Psychiatry. 2011; 50:1173-1185. [PubMed: 22024005]

50. Dickstein DP, et al. Cognitive flexibility in phenotypes of pediatric bipolar disorder. J Am Acad Child Adolesc Psychiatry. 2007; 46:341-355. [PubMed: 17314720]

51. White SF, et al. Dysfunctional representation of expected value is associated with reinforcementbased decision-making deficits in adolescents with conduct problems. J Child Psychol Psychiatry. 2016; 57:938-946. [PubMed: 27062170]

52. White SF, et al. Disrupted expected value and prediction error signaling in youths with disruptive behavior disorders during a passive avoidance task. Am J Psychiatry. 2013; 170:315-323. [PubMed: 23450288]

53. Finger EC, et al. Disrupted reinforcement signaling in the orbitofrontal cortex and caudate in youths with conduct disorder or oppositional defiant disorder and a high level of psychopathic traits. Am J Psychiatry. 2011; 168:152-162. [PubMed: 21078707]

54. White SF, et al. Disrupted expected value signaling in youth with disruptive behavior disorders to environmental reinforcers. J Am Acad Child Adolesc Psychiatry. 2014; 53:579-588. [PubMed: 24745957]

55. Séguin JR, et al. Cognitive and neuropsychological characteristics of physically aggressive boys. J Abnorm Psychol. 1995; 104:614-624. [PubMed: 8530764]

56. Séguin JR, et al. Executive functions and physical aggression after controlling for attention deficit hyperactivity disorder, general memory, and IQ. J Child Psychol Psychiatry. 1999; 40:1197-1208. [PubMed: 10604398]

57. Morgan AB, Lilienfeld SO. A meta-analytic review of the relation between antisocial behavior and neuropsychological measures of executive function. Clin Psychol Rev. 2000; 20:113-136. [PubMed: 10660831]

58. Suurland J, et al. Parental perceptions of aggressive behavior in preschoolers: Inhibitory control moderates the association with negative emotionality. Child Dev. 2016; 87:256-269. [PubMed: 26525924]

59. Granvald V, Marciszko C. Relations between key executive functions and aggression in childhood. Child Neuropsychol. 2015; 22:537-555. [PubMed: 25833167] 
60. Gagne JR, Goldsmith HH. A longitudinal analysis of anger and inhibitory control in twins from 12 to 36 months of age. Dev Sci. 2011; 14:112-124. [PubMed: 21159093]

61. Lamm C, et al. Magnitude and chronometry of neural mechanisms of emotion regulation in subtypes of aggressive children. Brain Cogn. 2011; 77:159-169. [PubMed: 21940093]

62. Rich BA, et al. Different psychophysiological and behavioral responses elicited by frustration in pediatric bipolar disorder and severe mood dysregulation. Am J Psychiatry. 2007; 164:309-317. [PubMed: 17267795]

63. Stringaris A, Goodman R. Mood lability and psychopathology in youth. Psychol Med. 2009; 39:1237-1245. [PubMed: 19079807]

64. Shaw P, et al. Emotional dysregulation in attention deficit hyperactivity disorder. Am J Psychiatry. 2014; 171:276-293. [PubMed: 24480998]

65. Karalunas SL, et al. Subtyping attention-deficit/hyperactivity disorder using temperament dimensions: Toward biologically based nosologic criteria. JAMA Psychiatry. 2014; 71:1015-1024. [PubMed: 25006969]

66. Sonuga-Barke EJ, et al. Annual Research Review: Transdiagnostic neuroscience of child and adolescent mental disorders--differentiating decision making in attention-deficit/hyperactivity disorder, conduct disorder, depression, and anxiety. J Child Psychol Psychiatry. 2016; 57:321-349. [PubMed: 26705858]

67. Hendricks MA, Buchanan TW. Individual differences in cognitive control processes and their relationship to emotion regulation. Cogn Emot. 2016; 30:912-924. [PubMed: 25947896]

68. Passarotti AM, et al. Emotion processing influences working memory circuits in pediatric bipolar disorder and attention-deficit/hyperactivity disorder. J Am Acad Child Adolesc Psychiatry. 2010; 49:1064-1080. [PubMed: 20855051]

69. Duarte NA, et al. Working memory deficits affect risky decision making in methamphetamine users with attention-deficit/hyperactivity disorder. J Psychiatr Res. 2012; 46:492-499. [PubMed: 22305489]

70. Yu X, et al. Preference for smaller sooner over larger later rewards in ADHD: contribution of delay duration and paradigm type. J Atten Disord. 2015; doi: 10.1177/1087054715570390

71. Costa Dias TG, et al. Reward circuit connectivity relates to delay discounting in children with attention-deficit/hyperactivity disorder. Eur Neuropsychopharmacol. 2013; 23:33-45. [PubMed: 23206930]

72. Wilbertz G, et al. Neural and psychophysiological markers of delay aversion in attention deficit hyperactivity disorder. J Abnorm Psychol. 2013; 122:566-572. [PubMed: 23713509]

73. Rich BA, et al. Different neural pathways to negative affect in youth with pediatric bipolar disorder and severe mood dysregulation. J Psychiatr Res. 2011; 45:1283-1294. [PubMed: 21561628]

74. Deveney CM, et al. Neural mechanisms of frustration in chronically irritable youth. Am $\mathbf{J}$ Psychiatry. 2013; 170:1186-1194. [PubMed: 23732841]

75. Pawliczek CM, et al. Anger under control: neural correlates of frustration as a function of trait aggression. PLoS One. 2013:e78503. [PubMed: 24205247]

76. Perlman SB, et al. fNIRS evidence of prefrontal regulation of frustration in early childhood. Neuroimage. 2014; 85:326-334. [PubMed: 23624495]

77. Perlman SB, et al. Neural substrates of child irritability in typically developing and psychiatric populations. Dev Cogn Neurosci. 2015; 14:71-80. [PubMed: 26218424]

78. Volkow ND, et al. Neurobiologic advances from the brain disease model of addiction. N Engl J Med. 2016; 374:363-371. [PubMed: 26816013]

79. Tovote P, et al. Neuronal circuits for fear and anxiety. Nat Rev Neurosci. 2015; 16:317-331. [PubMed: 25991441]

80. Insel TR, Cuthbert BN. Medicine. Brain disorders? Precisely Science. 2015; 348:499-500. [PubMed: 25931539]

81. Copeland WE, et al. Normative irritability in youth: Developmental findings from the great smoky mountains study. J Am Acad Child Adolesc Psychiatry. 2015; 54:635-42. [PubMed: 26210332] 
82. Blader JC, et al. Prevalence and treatment outcomes of persistent negative mood among children with attention-deficit/hyperactivity disorder and aggressive behavior. J Child Adolesc Psychopharmacol. 2016; 26:164-173. [PubMed: 26745211]

83. Copeland WE, et al. Prevalence, comorbidity, and correlates of DSM-5 proposed disruptive mood dysregulation disorder. Am J Psychiatry. 2013; 170:173-179. [PubMed: 23377638]

84. Wakschlag LS, et al. Defining the developmental parameters of temper loss in early childhood: implications for developmental psychopathology. J Child Psychol Psychiatry. 2012; 53:1099_ 1108. [PubMed: 22928674]

85. Wakschlag LS, et al. Clinical implications of a dimensional approach: The normal:abnormal spectrum of early irritability. J Am Acad Child Adolesc Psychiatry. 2015; 54:626-634. [PubMed: 26210331]

86. Gagne JR, Goldsmith H. A longitudinal analysis of anger and inhibitory control in twins from 12 to 36 months of age. Developmental Science. 2011; 14:112-124. [PubMed: 21159093]

87. He J, et al. Anger and approach motivation in infancy: Relations to early childhood inhibitory control and behavior problems. Infancy. 2010; 15:246-269. [PubMed: 25705134]

88. Leibenluft E, et al. Chronic vs. episodic irritability in youth: A community-based, longitudinal study of clinical and diagnostic associations. J Child Adolesc Psychopharmacol. 2006; 16:456466. [PubMed: 16958570]

89. Vidal-Ribas P, et al. The status of irritability in psychiatry: A conceptual and quantitative review. J Am Acad Child Adolesc Psychiatry. 2016; 55:556-570. [PubMed: 27343883]

90. Stringaris, et al. Adolescent irritability: Phenotypic associations and genetic links with depressed mood. Am J Psychiatry. 2012; 169:47-54. [PubMed: 22193524]

91. Roberson-Nay R, et al. Longitudinal stability of genetic and environmental influences on irritability: From childhood to young adulthood. Am J Psychiatry. 2015; 172:657-664. [PubMed: 25906668]

92. Savage J, et al. A genetically informed study of the longitudinal relation between irritability and anxious/depressed symptoms. J Am Acad Child Adolesc Psychiatry. 2015; 54:377-384. [PubMed: 25901774]

93. Kaminski JW, et al. A meta-analytic review of components associated with parent training program effectiveness. J Abnorm Child Psychol. 2008; 36:567-589. [PubMed: 18205039]

94. Stoddard J, et al. Irritability in child and adolescent anxiety disorders. Depress Anxiety. 2014; 31:566-573. [PubMed: 23818321]

95. Dodge KA, Coie JD. Social-information-processing factors in reactive and proactive aggression in children's peer groups. J Pers Soc Psychol. 1987; 53:1146-1158. [PubMed: 3694454]

96. Baskin-Sommers AR, et al. Characterizing the anomalous cognition-emotion interactions in externalizing. Biol Psychol. 2012; 91:48-58. [PubMed: 22579718] 


\section{Trends Box}

- Irritability in youth is a common clinical problem that may result from aberrant responses to frustration and/or aberrant approach responses to threat.

- Irritability and anxiety are associated cross-sectionally, longitudinally, and genetically. These associations suggest that disequilibrium in threat response circuitry is important in the pathophysiology of irritability.

- $\quad$ Studies using simple or complex social stimuli find hyper-reactivity to threat in irritable youth, mediated by dysfunction in the amygdala, medial prefrontal cortex, ACC, insula, striatum, and association cortex.

- $\quad$ Studies find reward learning abnormalities in irritable youth, as well as cognitive control deficits and exaggerated responses on paradigms modeling frustration. Such abnormalities are associated with dysfunction in regions mediating reward learning (e.g., inferior frontal gyrus, striatum, ventromedial prefrontal cortex) and cognitive control and attention (e.g., anterior cingulate cortex, parietal cortex).

- Pathophysiological studies of irritability are enabling the development of mechanism-based, well-targeted, effective interventions. 


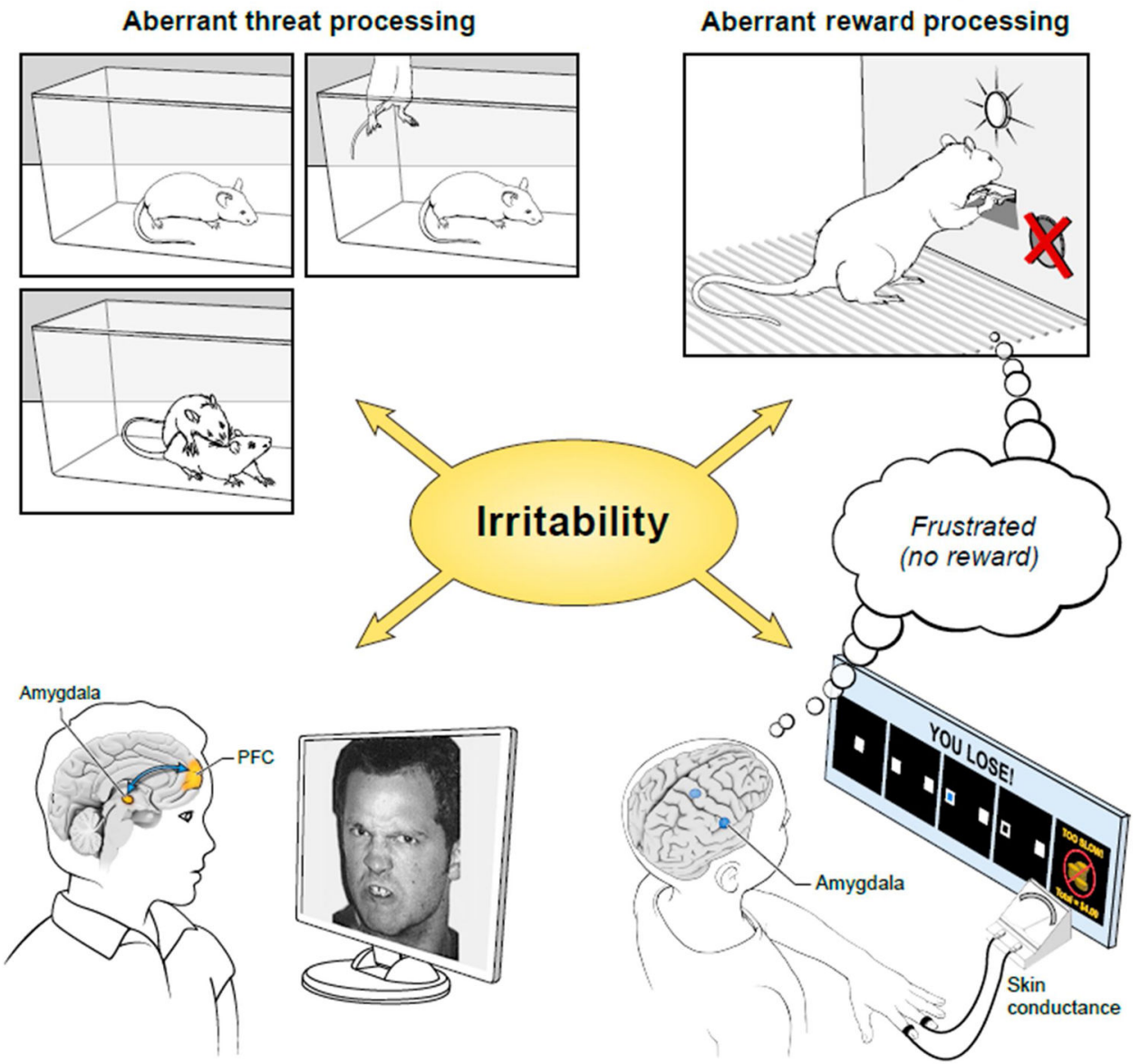

Figure 1 (Key Figure). Irritability in rodents and humans is associated with aberrant responses to threat and/or frustrative non-reward

The figure shows parallel processes that can be observed in rodents and humans. Drawings on the left show associations between aberrant responses to threat and irritability in both species. Experimental manipulations that expose an organism to threat can be used to probe this pathway to irritability. Top left: When exposed to the threat of a conspecific in a resident intruder paradigm, frustrated mice show increased aggressive behavior. This aggression is greater than that exhibited by non-frustrated mice (not shown) [12]. Bottom left: Irritability in youth is associated with aberrant amygdala-prefrontal cortex connectivity when viewing threatening faces [37]; see Figure 2 for details. Drawings on the right show associations between aberrant responses to frustration and irritability in in both species. Experimental manipulations that expose an organism to threat can be used to study between-strain or between-subject differences in response to frustration, in the form of irritable behavior. Top right: Frustration is induced in a mouse by withholding food reward when the appearance of the conditioned stimulus (light cue) signals the opportunity to obtain reward through lever press [12]. Bottom right: Frustration is induced in a child by a game that is rigged so that the child does not receive an expected reward [74]; see also Figure 3. 


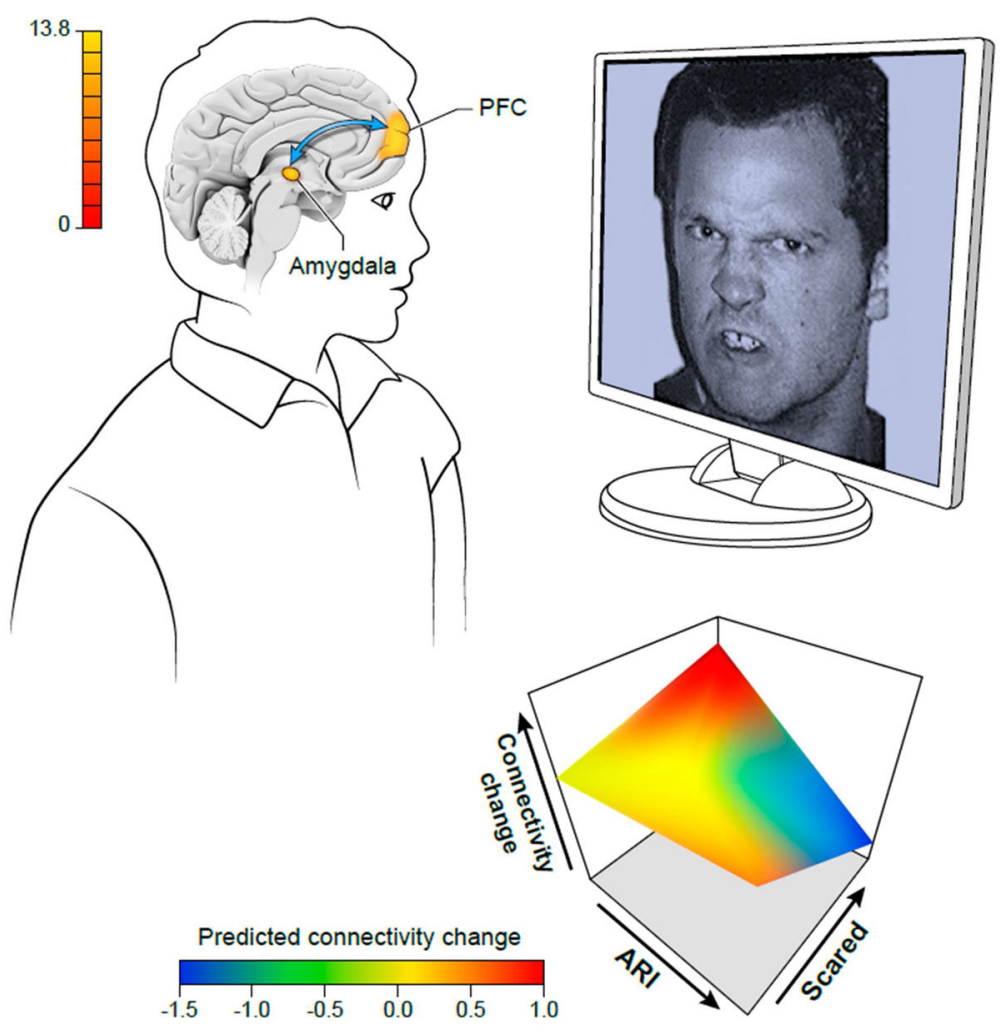

Figure 2. Amygdala-prefrontal cortex connectivity is driven by an interaction between levels of anxiety and levels of irritability when youth view a threatening face

For details, see Stoddard et al. [37]. Briefly summarized, fMRI data were obtained while youth (ages 8-17 y, n=93 with anxiety, DMDD and/or ADHD, 22 healthy volunteers)

performed an implicit face emotion processing task (i.e., gender identification) on angry, happy, and fearful faces at 50\%, 100\%, and $150 \%$ intensity. Functional connectivity was examined using a psychophysiological interaction analysis with an amygdala seed. When subjects viewed a $150 \%$ angry face, amygdala connectivity was related to levels of irritability, anxiety, and their interaction. As illustrated in the three dimensional plane, an interaction between irritability (measured on the ARI scale) and anxiety (measured on the Scared scale) was found for amygdala-medial prefrontal cortex connectivity. Specifically, decreasing connectivity was associated with increasing levels of both irritability and anxiety, while increasing connectivity was associated with increasing anxiety in the absence of increasing irritability. 


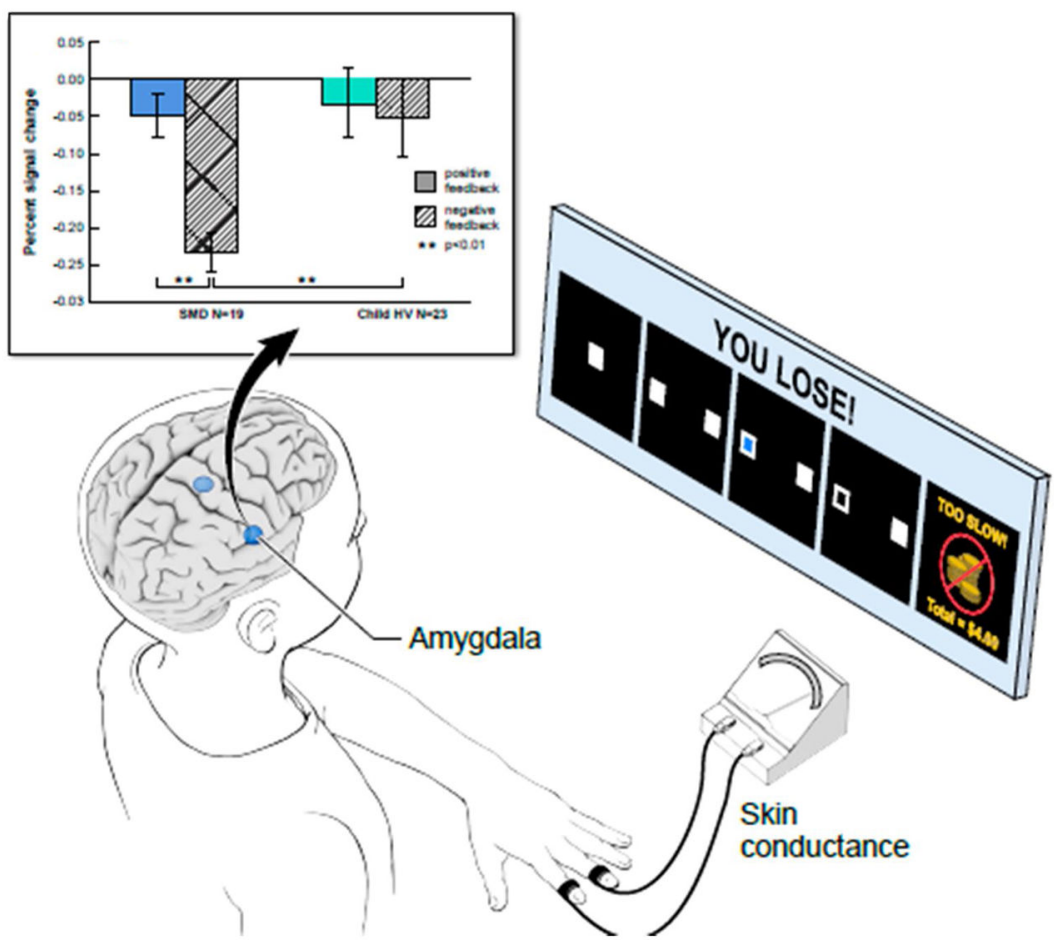

Figure 3. Amygdala de-activation differs between chronically irritable youth and healthy volunteers while playing a frustrating game

Frustration is induced in children by first inducing reward expectation i.e., children play a game in which they can easily earn a reward (non-frustration condition). This non-frustration condition is then followed by a frustration condition, during which the game is rigged so that the child no longer receives the reward he has come to expect. Physiological measures such as skin conductance and BOLD fMRI signal can be measured while the child plays. The data here are from an fMRI study in which youth with severe mood dysregulation (SMD, the research precursor to DMDD) and healthy youth played a frustrating game. During the frustration condition, youth with SMD showed decreased amygdala activity when receiving frustrating, negative feedback ("you lose"). For details, see Deveney et al. [74]. 